\title{
Face Recognition Technique Using Symbolic Linear Discriminant Analysis Method
}

\author{
P.S. Hiremath ${ }^{1}$ and Prabhakar C.J. ${ }^{2}$ \\ ${ }^{1}$ Department of Studies in Computer Science, Gulbarga University, \\ Gulbarga - 585106, Karnataka, India \\ hiremathps@yahoo.co.in \\ ${ }^{2}$ Department of Studies in Computer Science, Kuvempu University, \\ Shankaraghatta - 577451, Karnataka, India \\ psajjan@yahoo.com
}

\begin{abstract}
Techniques that can introduce low dimensional feature representation with enhanced discriminatory power are important in face recognition systems. This paper presents one of the symbolic factor analysis method i.e., symbolic Linear Discriminant Analysis (symbolic LDA) method for face representation and recognition. Classical factor analysis methods extract features, which are single valued in nature to represent face images. These single valued variables may not be able to capture variation of each feature in all the images of same subject; this leads to loss of information. The symbolic Linear Discriminant Analysis Algorithm extracts most discriminating interval type features; they optimally discriminate among the classes represented in the training set. The proposed method has been successfully tested for face recognition using two databases, ORL and Yale Face database. The effectiveness of the proposed method is shown in terms of comparative performance against popular classical factor analysis methods such as eigenface method and Linear Discriminant Analysis method. Experimental results show that symbolic LDA outperforms the classical factor analysis methods.
\end{abstract}

\section{Introduction}

Feature extraction has been the most fundamental and important in face recognition and other recognition problems. The main objective of research on face recognition problem is to find a technique that can introduce low dimensional feature representation of face objects with enhanced discriminatory power. Among various solutions to this problem, the most successful are those appearance-based approaches [4][5][19][26][27]. Principal Component Analysis (PCA) is a well known appearance based technique, Kirby and Sirovich [14] are among the first who used this technique directly on the characterization of human faces and showed that PCA is an optimal compression scheme that minimizes the mean squared error between the original images and their reconstructions for any given level of compression. Turk and Pentland [22] popularized the use of PCA for face recognition; they used PCA to compute a set of subspace basis vectors (which they called eigenfaces) for a database of face 
images. Grudin [11] showed that the correlation between images of the whole faces is not efficient for satisfactory recognition performance. Illumination normalization is usually necessary for the eigenface approach. Zhao and Yang proposed a new method to compute the covariance matrix using three images each taken in different lighting conditions to account for arbitrary illumination effects. Bartlett et al., [2] proposed using Independent Component Analysis (ICA) for face representation and found that it was better than PCA when cosines were used as the similarity measure. ICA improves upon the PCA scheme by considering higher order statistics and ICA searches for a linear transformation to express a set of random variables as linear combinations of statistically independent source variables. Yang [24] used Kernel PCA for face feature extraction and recognition and showed that the kernel eigenfaces method outperforms the classical eigenfaces method. However, ICA and Kernel PCA are both computationally more expensive than PCA. Swets and Weng [21] present a method to selecting discriminant eigenfeatures using multidimensional linear discriminant analysis (LDA). LDA [25] based algorithms outperform PCA based ones, since the former optimizes the low-dimensional representation of the objects with focus on the Most Discriminant Features (MDFs) extraction while the latter achieves simply object reconstruction. PCA based technique produce a set of Most Expressive Features (MEFs); the features produced are not necessarily good for discriminating among classes defined by the set of samples. The MEFs describe some major variations in the class, such as those due to lighting direction; these variations may well be irrelevant to how the classes are divided. Recently, many appearance-based algorithms have been proposed [9][16][17][18][23][24][27].

The defining characteristic of these classical appearance-based algorithms is that they directly use the pixel intensity values in a face image as the features on which to base the recognition decision. The pixel intensities that are used as features are represented using single valued variables. However, in many situations same face is captured under different orientations, lighting conditions, expressions and backgrounds, which lead to image variations. The corresponding pixel intensities do change because of image variations. The use of single valued variables may not be able to capture the variation of feature values of the images of the same subject. In such a case, we need to consider the symbolic data analysis (SDA) [1][8][15], in which the interval-valued data are analyzed. We have focused our research towards extracting interval type features to represent face images, which are robust to variations due to illumination, orientation and facial expression. In [12], a Symbolic Principal Component Analysis (symbolic PCA) approach for face recognition is presented, in which symbolic PCA is employed to compute a set of subspace basis vectors for symbolic faces and then project the symbolic faces into the compressed subspace. This method requires a small number of features to achieve the same recognition rate as compared to eigenface method. The symbolic PCA technique, however, encodes only for second order statistics, i.e., pixel wise covariance among the pixels, and does not address high-order statistical dependencies such as the relationships among three or more pixels. As these second order statistics provide only partial information on the statistics of both natural images and human faces, it might become necessary to incorporate 
higher order statistics as well. The kernel PCA [20][24] is capable of deriving low dimensional features that incorporate higher order statistics. Higher order dependencies in an image include nonlinear relations among the pixel intensity values, such as the relationships among three or more pixels in an edge or a curve, which can capture important information for recognition. The kernel PCA is extended to symbolic data analysis as symbolic KPCA [13] for face recognition and the experimental results show improved recognition rate as compared to the symbolic PCA method.

In this paper, symbolic LDA method is presented, which is generalization of the classical linear discriminant analysis to symbolic objects for face recognition. In the first phase, we represent the face images as symbolic objects (symbolic faces) of interval type variables. The representation of face images as symbolic faces accounts for image variations of human faces under different lighting conditions, orientations and facial expressions. It also drastically reduces the dimension of the image space without losing a significant amount of information. Each symbolic face summarizes the variation of feature values through the different images of the same subject. In the second phase, we have applied the proposed symbolic LDA on the symbolic faces and linearly derives low dimensional most discriminant interval type features. Finally, a minimum distance classifier with Minkowsky's symbolic dissimilarity measure proposed by De Carvalho and Diday [1] is employed for classification. The proposed method has been successfully tested using two standard databases ORL and Yale Face database.

The remainder of this paper is organized as follows: the construction of symbolic faces is described in section 2. In section 3, extraction of most discriminant interval type features from symbolic faces is presented. The comparison results are presented in the section 4. Finally, a conclusion is drawn in the section 5.

\section{Extraction of Symbolic Faces}

Let $\Omega=\left\{\Gamma_{1}, \ldots, \Gamma_{n}\right\}$ be the collection of $n$ face images of the database, each of size $N \times M$. An image set is a collection of face images of $m$ different subjects (face class) denoted by $E=\left\{c_{1}, c_{2}, \ldots, c_{m}\right\}$. We have assumed that images belonging to a face class are arranged from right side view to left side view. The view range of each face class is partitioned into $q$ sub face classes and each sub face class contains $r$ number of images. The feature vector of $k^{\text {th }}$ sub face class $c_{i}^{k}$ of $i^{\text {th }}$ face class $c_{i}$, where $k=1,2, \ldots, q$, is described by a vector of $p$ interval variables $Y_{1}, \ldots, Y_{p}$, and is of length $p=N M$. The interval variable $Y_{j}$ of $k^{\text {th }}$ sub face class $c_{i}^{k}$ of $i^{\text {th }}$ face class is described as $Y_{j}\left(c_{i}^{k}\right)=\left[\underline{x}_{i j}^{k}, x_{i j}^{k}\right]$, where $\underline{x}_{i j}^{k}$ and $\bar{x}_{i j}^{k}$ are minimum and maximum intensity values, respectively, among $j^{\text {th }}$ pixels of all the images of sub face class $c_{i}^{k}$. The vector $X_{i}^{k}$ of interval variables is recorded for $k^{\text {th }}$ sub face class $c_{i}^{k}$ of $i^{\text {th }}$ face class. This vector is called as symbolic face. 
We denote

$$
X_{i}^{k}=\left(Y_{1}\left(c_{i}^{k}\right), \ldots, Y_{p}\left(c_{i}^{k}\right)\right), i=1, \ldots, m, k=1, \ldots, q, j=1, \ldots, p .
$$

We represent the $q m$ symbolic faces by a matrix $X$ of size $(p \times q m)$, consisting of column vectors $X_{i}^{k}, i=1, \ldots, m, k=1, \ldots, q$.

\section{Extraction of Most Discriminant Interval Type Features}

Let us consider the matrix $X$ containing $q m$ symbolic faces pertaining to the given set $\Omega$ of images belonging to $m$ face classes. The centers [6] [7] $x_{i j}^{k} \in \Re$ of the intervals $\left[\underline{x}_{i j}^{k}, x_{i j}^{k}\right]$, are given by:

$$
x_{i j}^{k^{c}}=\frac{\bar{x}_{i j}^{k}+\underline{x}_{i j}^{k}}{2}
$$

where $i=1, \ldots, m$ and $j=1, \ldots, p$.

The $p \times q m$ data matrix $X^{C}$ containing the centers $x_{i j}^{k^{c}} \in \mathfrak{R}$ of the intervals for $q m$ symbolic faces. The $p$-dimensional vectors $X_{i}^{{ }^{C}}=\left(x_{i 1}^{k}, \ldots, x_{i p}^{k}\right)$, $\underline{X}_{i}^{k}=\left(\underline{x}_{i 1}^{k}, \ldots, \underline{x}_{i p}^{k}\right)$ and $\bar{X}_{i}^{k}=\left(\bar{x}_{i 1}^{k}, \ldots, \bar{x}_{i p}^{k}\right)$ represent the centers, lower bounds and upper bounds of the $q m$ symbolic faces $X_{i}^{k}$, respectively. There are $m$ face classes denoted by $E=\left\{c_{1}, c_{2}, \ldots, c_{m}\right\}$, each class $c_{i}$ contains $q_{i}, i=1,2, \ldots, m$, number of symbolic faces.

The mean $M_{i}$ of class $c_{i}$ is calculated as

$$
M_{i}=\frac{1}{q_{i}} \sum_{k=1}^{q_{i}} X_{i}{ }^{C}
$$

where $q_{i}$ is number of symbolic faces of class $c_{i}$.

Similarly the grand mean vector of all $q m$ symbolic faces from all classes is defined as

$$
M=\frac{1}{q m} \sum_{L=1}^{q m} X_{i}^{k}, \text { for } i=1, \ldots, m, \text { and } k=1, \ldots, q .
$$

The within class scatter matrix $S_{w}$ is defined as

$$
S_{w}=\sum_{i=1}^{m} \sum_{k=1}^{q_{i}}\left[\left(X_{i}^{k^{C}}-M_{i}\right)^{T}\right]\left[\left(X_{i}^{k^{C}}-M_{i}\right)\right]
$$

The between class scatter matrix $S_{b}$ is defined as 


$$
S_{b}=\sum_{i=1}^{m}\left[\left(M_{i}-M\right)^{T}\right]\left[\left(M_{i}-M\right)\right]
$$

where $M_{i}$ is mean of class $c_{i}(i=1, \ldots, m)$.

In discriminant analysis, we want to determine the projection axis $W$ that maximizes the ratio $\frac{\operatorname{det}\left\{S_{b}\right\}}{\operatorname{det}\left\{S_{w}\right\}}$. In other words, we want to maximize the between-class scatter matrix while minimizing the within-class scatter matrix. It has been proven (Fisher and Loeve) [10] that this ratio is maximized when the column vectors of projection axis $W$ is the eigenvector of $S_{w}^{-1} S_{b}$ associated with the largest eigenvalue.

We need a set of projection axes, $W_{1}, \ldots, W_{d}$, which are eigenvectors of $S_{w}^{-1} S_{b}$ corresponding to first $d$ largest eigenvalues. The optimal discriminant projection axes, $W_{1}, \ldots, W_{d}$ are used for feature extraction.

Since, each symbolic face $X_{i}^{k}$ is located between the lower bound symbolic face $\underline{X}_{i}^{k}$ and upper bound symbolic face $\bar{X}_{i}^{k}$, so it is possible to find most discriminating interval type features $\left[\underline{B_{i}^{k}}, \bar{B}_{i}^{k}\right]$.

The lower bound features of each symbolic face $X_{i}^{k}$ is given by

$$
\underline{B}_{i}^{k}=W_{l}^{T} \underline{X}_{i}^{k} \quad, l=1,2, \ldots, d
$$

for $i=1, \ldots, m, k=1, \ldots, q$.

Similarly the upper bound features of each symbolic face $X_{i}^{k}$ is given by

$$
\bar{B}_{i}^{k}=W_{l}^{T} \bar{X}_{i}^{k}, l=1,2, \ldots, d
$$

Let $c_{\text {test }}=\left[\Gamma_{1}, \Gamma_{2}, \ldots, \Gamma_{l}\right]$ be the test face class contains face images of same subject with different expression, lighting condition and orientation. The test symbolic face $X_{\text {test }}$ is constructed for test face class $c_{\text {test }}$ as explained in the section 2 . The lower bound test symbolic face of test symbolic face $X_{\text {test }}$ is described as $\underline{X}_{\text {test }}=\left(\underline{x}_{1}^{\text {test }}, \underline{x}_{2}^{\text {test }}, \ldots, \underline{x}_{p}^{\text {test }}\right)$. Similarly, the upper bound test symbolic face is described as $\bar{X}_{\text {test }}=\left(\begin{array}{ll}\bar{x}_{\text {test }} & -\bar{x}_{\text {test }}, \ldots, \bar{x}_{p}^{\text {test }} \\ x_{1}\end{array}\right)$.

The interval type features $\left[\underline{B}^{\text {test }}, \bar{B}^{\text {test }}\right]$ of test symbolic face $X_{\text {test }}$ are computed as:

$$
\begin{gathered}
\underline{B}^{\text {test }}=W_{l}^{T} \underline{X}_{\text {test }}, \\
\bar{B}^{\text {test }}=W_{l}^{T} \bar{X}_{\text {test }},
\end{gathered}
$$

where $l=1,2, \ldots, d$. 


\subsection{Face Recognition Based on a Minimum Distance Classifier}

When test face class $c_{\text {test }}$ is presented to the symbolic LDA classifier, low dimensional interval features $\left[\underline{B}^{\text {test }}, \bar{B}{ }^{\text {test }}\right]$ are derived. Let, $\left[\underline{B}_{i}^{k}, \bar{B}_{i}^{k}\right]$, $i=1, \ldots, m, k=1, \ldots, q$, be the interval features of $q m$ symbolic faces. The classifier applies the minimum distance rule for classification using symbolic dissimilarity measure $\delta$ :

$\delta\left(\left[\underline{B}^{\text {test }}, \bar{B}^{\text {test }}\right],\left[\underline{B}_{i}^{k}, \bar{B}_{i}^{k}\right]\right)=\min _{i} \delta\left(\left[\underline{B}^{\text {test }}, \bar{B}^{\text {test }}\right],\left[\underline{B}_{i}^{k}, \bar{B}_{i}^{k}\right]\right) \rightarrow c_{\text {test }} \in c_{i}$

The symbolic LDA interval feature vector $\left[\underline{B}^{\text {test }}, \bar{B}{ }^{\text {test }}\right]$ is classified as belonging to the symbolic face, $c_{i}$, using appropriate symbolic dissimilarity measure $\delta$.

\section{Experimental Results}

Face recognition system using symbolic LDA method identify the face by computing nearest face image for a given unknown face images using minimum distance classification rule. If our system is able to recognize given probe image is to same face image in the database then the trial is success, otherwise it is a failure. To show the significance symbolic LDA for dimensionality reduction we have chosen different sized subspaces for experiments. We assess the feasibility and performance of the proposed symbolic LDA on the face recognition task, using ORL and Yale databases, these databases contains frontal face images which were acquired under variable illumination, orientation and facial expressions. The effectiveness of proposed methods is shown in terms of comparative performance against five popular face recognition methods. In particular, we compared our algorithms with eigenfaces [22], fisherfaces [3], symbolic PCA [12], symbolic ICA and symbolic KPCA [13]. The experimentation is done on system with CPU: Pentium $2.5 \mathrm{GHz}$.

\subsection{Experiments Using ORL Database}

We assess the feasibility and performance of the proposed symbolic LDA on the face recognition task using ORL database. The ORL face database is composed of 400 images with ten different images for each of the 40 distinct subjects. All the images were taken against a dark homogeneous background with the subjects in an upright, frontal position, with tolerance for some tilting and rotation of up to about $20^{\circ}$ from frontal view to left side view and right side view. There is some variation in scale of up to about $10 \%$. The spatial and gray level resolutions of the images are $92 \times 112$ and 256, respectively. In our experiments, based on the eye positions, all the face images in the database and the query input are manually cropped to a size of $80 \times 80$. All the 400 images from the ORL database are used to evaluate the face recognition performance of proposed methods. We have manually arranged the face images of 
same subject from right side view to left side view. Six images are randomly chosen from the ten images available for each subject for training, while the remaining images are used to construct the test symbolic face for each trial. Fig.1 shows the some typical images of one subject of ORL database.

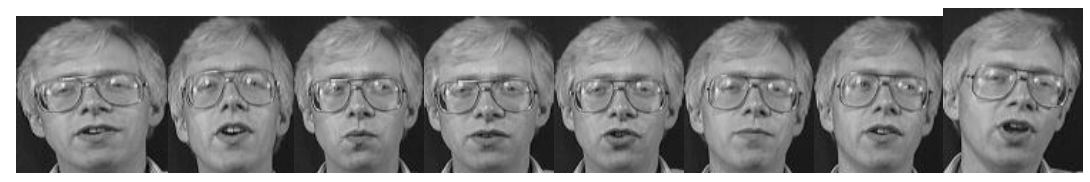

Fig. 1. Sample face images belonging to one subject of ORL database

Table 1. Comparison of classification performance of proposed symbolic LDA method using ORL database

\begin{tabular}{|l|c|c|c|}
\hline \multicolumn{1}{|c|}{ Methods } & Training time $(\mathrm{sec})$ & Feature Dimension & Recognition Rate (\%) \\
\hline Fisherfaces & 98 & 86 & 92.8 \\
\hline Eigenfaces & 102 & 189 & 87.65 \\
\hline Symbolic PCA & 38 & 71 & 94.85 \\
\hline Symbolic ICA & 87 & 109 & 89.15 \\
\hline Symbolic KPCA & 110 & 49 & 95.45 \\
\hline Symbolic LDA & 19 & 28 & 97.5 \\
\hline
\end{tabular}

Table-1 presents the experimental results for each method corresponding to ORL database. The experimental results show that the proposed method outperforms the classical factor analysis methods. Further, the symbolic LDA method achieves the better recognition rate than symbolic PCA method, symbolic ICA and symbolic KPCA method.

\subsection{Experiments on the Yale Face Database}

The experiments are conducted using Yale database to evaluate the excellence of the symbolic LDA for the face recognition problem. The Yale Face database consists of a total 165 images obtained from 15 different people, with 11 images from each person. The images contain variations in the following facial expressions or configurations: center-light, with glasses, happy, left light, without glasses, normal, right light, sad, sleepy, surprised and wink. We preprocessed these images by aligning and scaling them so that the distances between the eyes were the same for all images and also ensuring that the eyes occurred in the same co-ordinates of the image. The resulting image was then cropped. The final image was $128 \times 156$. The Fig. 2 shows some typical images of one subject of Yale Face database.

In our experiments, 9 images are randomly chosen from each class for training, while the remaining two images are used to construct test symbolic face for each trial. The recognition rates, training time and optimal subspace dimension are listed in Table-2. From Table-2, we note that the symbolic LDA method with a smaller number of features outperforms the classical factor analysis methods with a larger number of features. 


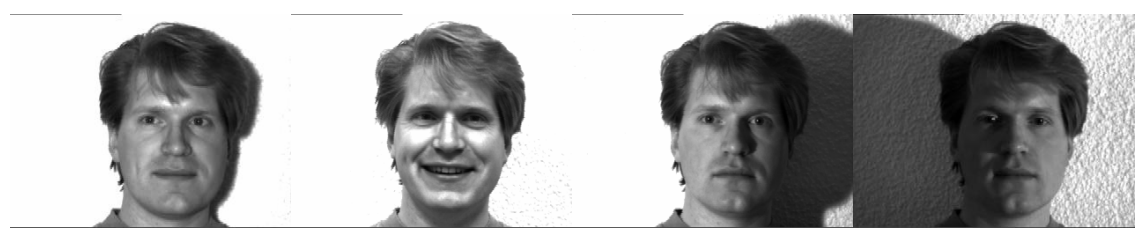

Fig. 2. Some typical images of one subject of Yale Face database

Table 2. Comparison of classification performance using Yale Face database

\begin{tabular}{|l|c|c|c|}
\hline \multicolumn{1}{|c|}{ Methods } & Training time $(\mathrm{sec})$ & Feature Dimension & Recognition Rate (\%) \\
\hline Fisherfaces & 59 & 23 & 89.85 \\
\hline Eigenfaces & 85 & 110 & 82.04 \\
\hline Symbolic PCA & 35 & 41 & 91.15 \\
\hline Symbolic ICA & 43 & 32 & 92.00 \\
\hline Symbolic KPCA & 98 & 56 & 94.55 \\
\hline Symbolic LDA & 18 & 13 & 96.15 \\
\hline
\end{tabular}

\section{Conclusion}

This paper presents symbolic LDA for face recognition. The feasibility of the symbolic LDA method has been tested successfully using standard databases, ORL and Yale Face database. The experimental results show that the proposed method achieve significantly improved recognition rates as compared to classical factor analysis methods. The proposed symbolic LDA outperforms symbolic PCA, symbolic ICA and symbolic KPCA under variable lighting conditions, orientations and expressions.

The proposed symbolic LDA has many advantages compared to classical factor analysis methods. The drawback of classical factor analysis methods is that in order to recognize a face seen from a particular pose and under a particular illumination, the face must have been previously seen under the same conditions. The symbolic LDA overcomes this limitation by representing the faces by interval type features so that even the faces seen previously in different poses, orientations and illuminations are recognized. Another important merit is that we can use more than one probe images with inherent variability of a face for face recognition. Therefore, symbolic LDA improve the recognition accuracy as compared to classical factor analysis methods at reduced computational cost. This is clearly evident from the experimental results. Further, the symbolic LDA yields significantly better results than other symbolic factor analysis methods.

\section{References}

1. Bock, H.H., Diday, E. (Eds.) 2000. Analysis of Symbolic Data. Springer Verlag.

2. Bartlett, Lades, Sejnowski: Independent component representation for face recognition, In: Proc. of the SPIE (1998) 528-539.

3. P. Belhumeur, J. Hespanha, D. Kriegman,1997. Eigenfaces vs. Fisherfaces: Recognition Using Class Specific Linear Projection. IEEE Transaction on PAMI.19 (7): 711-720. 
4. Bruneli and Poggio: Face Recognition: Features versus Templates, vol -15. IEEE Trans. Pattern Analysis and Machine Intelligence, (1993)1042-1052.

5. Chellappa, Wilson, Sirohey, 1995. Human and machine recognition of faces: A survey, vol - 83(5). Proc.IEEE) 705-740,

6. Choukria, Diday, Cazes, 1995. Extension of the principal component analysis to interval data. Presented at NTTS'95: New Techniques and Technologies for statistics, Bonn.

7. Choukria, Diday, Cazes, 1998. Vertices Principal Component Analysis with an Improved Factorial Representation. In: A. Rizzi, M.Vichi, H.Bock (eds.): Advances in Data Science and Classification. Pp.397-402, Springer Verlag .

8. Diday, 1993. An Introduction to symbolic data analysis. Tutorial at IV Conf. IFCS.

9. K.Etemad and R.Chellappa, 1997. "Discriminant Analysis for Recognition of Human Face Images", J.Optical Soc. Am. vol-14, pp 1724-1733.

10. Fisher, 1938. The statistical utilization of multiple measurements, Ann. Eugenics, 8, 376-386.

11. M.A.Grudin, 2000. "On internal representations in face recognition systems", Pattern recognition, vol.33, no.7, pp.1161-1177.

12. Hiremath.P.S, Prabhakar.C.J, 2005. "Face Recognition Technique using Symbolic PCA Method", Proc. Int. Conf. on Pattern Recognition and Machine Intelligence (PreMI'05), Kolkata, 266-271, Springer Verlag.

13. Hiremath.PS, Prabhakar.C.J, "Face Recognition Technique using Symbolic kernel PCA Method”, Proc. Int. Conf. on Cognition and Recognition (COGREC'05), Mysore, 801-805, Allied Publishers (2005).

14. Kirby, Sirovich, 1990. Applications of the Karhunen-Loeve procedure for the characterization of human faces, v-12(1). IEEE Trans. Pattern Anal. Machine Intell. 103-108.

15. Lauro, Verde, Palumbo, 1997. Analysis of symbolic data, Bock and Diday (Eds), Springer Verlag.

16. Liu, Wechsler, , 2002. Gabor feature based classification using the enhanced Fisher linear discriminant model for face recognition. IEEE Trans. Image Process. 11(4), 467-476.

17. Liu, Wechsler, 2000: Robust coding schemes for indexing and retrieval from large face databases, IEEE Trans. On image processing 9, 132-137.

18. Liu, Cheng, Yang, 1993. Algebraic feature extraction for image Recognition based on an optimal discriminant criterion, Pattern Recognition, 26, 903-911.

19. A.Pentland, B.Moghaddam and T.Starner, 1994. "View based and modular Eigenfaces for Face Recognition", Proc. Computer Vision and Pattern Recognition, pp.84-91.

20. Scholkopf and A.Smola, and K.Muller, "Nonlinear Component Analysis as a kernel Eigenvalue Problem", Neural Computation, vol.10, pp.1299-1319, 1998.

21. D.Swets, J.Weng, 1996. Using discriminant eigenfeatures for image retrieval. IEEE. Transactions on PAMI,18, 831-836.

22. Turk, Pentland, 1991. Eigenfaces for Recognation, v-3. J Cognitive Neuro Science, 71-86.

23. Yu, Yang, 2001. A Direct LDA algorithm for high dimensional data with application to face recognition, Pattern Recognition, 34(7), 2067-2070.

24. M.H.Yang, "Kernel Eigenfaces vs. Kernel Fisherfaces:Face Recognition using Kernel Methods", Proc. Fifth IEEE Int'l Conf. Automatic Face and Gesture Recognition,pp.215220,2002.

25. M.H.Yang, N.Ahuja and D.Kriegman, “Face Recognition Using Kernel Eigenfaces”, Proc. IEEE Int'l Conf. Image Processing, 2000.

26. Zhao, Chellappa, Phillips, 1999. Subspace linear discriminant analysis for face recognition. Technical Report, CS-TR4009, University of Maryland.

27. Zhao, Chellappa, Phillips, Rosenfeld, 2003. Face Recognition: A literature survey, ACM Comput. Surveys, 35(4), 399-458. 